\title{
Des liens intergénérationnels à la transmission transgénérationnelle : vers une co-création marque-consommateur
}

Étude du marketing de la nostalgie de Danette

Daphné Duvernay

\section{OpenEdition}

Journals

Édition électronique

URL : http://journals.openedition.org/communicationorganisation/3526

DOI : 10.4000/communicationorganisation.3526

ISSN : $1775-3546$

Éditeur

Presses universitaires de Bordeaux

Édition imprimée

Date de publication : 1 décembre 2011

Pagination : $37-58$

ISBN : 978-2-86781-745-8

ISSN : 1168-5549

Référence électronique

Daphné Duvernay, « Des liens intergénérationnels à la transmission transgénérationnelle : vers une cocréation marque-consommateur », Communication et organisation [En ligne], 40 | 2011, mis en ligne le 01 décembre 2014, consulté le 19 avril 2019. URL : http://journals.openedition.org/ communicationorganisation/3526 ; DOI : 10.4000/communicationorganisation.3526 


\title{
Des liens intergénérationnels à la transmission transgénérationnelle : vers une co-création marque-consommateur Étude du marketing de la nostalgie de Danette
}

\author{
Daphné Duvernaỵ'
}

Nous inscrivons la problématique de cette présente contribution surl'analyse des pratiques de communication générationnelle appréhendées comme discours et constructions symboliques produits par l'organisation. L'approche communicationnelle des organisations (Bouillon et al., 2007) permet d'opérer un "grand écart épistémologique " pour appréhender, ensemble, l’individuel et le collectif en établissant des liens entre des disciplines voisines mais différentes. Il s'agit de montrer ici, comment les acteurs construisent du sens à travers les actions qu'ils conduisent, mettant en œuvre des " effets de génération " au sein d'époques marquées par des évolutions technologiques, médiatiques et socioculturelles. Ces évolutions peuvent se produire grâce aux actions et aux discours qui les portent. Cette vision s’inscrit dans une perspective constructiviste au sein de laquelle « la signification est avant tout construite, établie hic et nunc entre sujets qui se construisent dans toute relation signifiante, construisent leur objet et se construisent à travers lui » (Boutaud, 1998).

À partir de l'étude du cas Danette, nous voulons montrer la transition d'une génération à l'autre, comment cette communication médiatique a engendré un basculement d'une "verticalité des pères » à une " horizontalité des pairs ». À travers cette question générationnelle, se pose une évolution d'une économie de la transaction à une économie de la relation, où pour demeurer compétitives, les entreprises doivent considérer le « cycle de vie client » plutôt que le « cycle de vie produit ». Aujourd'hui, d'un point de vue gustatif et qualitatif, la différence entre les produits Danette et ceux des marques de

1. Daphné Duvernay est Maître de Conférences en Sciences de l'Information et de la Communication à I'Université du Sud Toulon Var. Elle est chercheure au sein du laboratoire I3M. Ses thématiques de recherche portent sur la communication de changement dans les organisations, les nouvelles pratiques d'informations et de communication sur l'internet et les réseaux socionumériques ; duvernay@univ-tln.fr 
distributeurs reste marginale, si ce n'est celle du prix. L'avantage concurrentiel de Danone se jouera sur un « ré-enchantement » consumériste, par la mise en œuvre d'une communication relationnelle et personnalisée.

Dans une première partie, nous proposons une approche allagmatique en SIC des notions d'âge et de génération (Carayol, 2004). Après avoir décrit notre méthodologie d'analyse de l'étude de cas Danette dans une deuxième partie, nous présentons nos principaux résultats dans les deux parties suivantes. Nous montrons comment Danone est parvenu à construire, à partir de l'histoire de Danette, un imaginaire sociotechnique (Flichy, 2001) de la communication générationnelle de la marque. Nous nous centrons ensuite plus spécifiquement sur le «travail du consommateur » (Dujarier, 2008) à travers l'appel à contribution que Danette met en œuvre auprès des internautes afin de les associer étroitement à la production, à la commercialisation et à la médiatisation des produits Danette. Enfin, nous terminons par une discussion allagmatique de l'ensemble de ces résultats pour retracer l'évolution du marketing de la nostalgie déployé par la marque, depuis les liens intergénérationnels jusqu’à la transmission transgénérationnelle.

\section{La segmentation générationnelle : une lecture communicationnelle allagmatique des organisations}

D'abord centrées, depuis les années 1970, sur les caractéristiques intrinsèques du produit, les marques ont dû évoluer sur leur registre discursif à destination de leurs consommateurs. Cette évolution s'est faite dans les années 1990, où la nécessité d'une profonde compréhension du consommateur se fait sentir. Les marques délaissent une communication basée sur les médias de masse pour une communication plus relationnelle, via les database marketing (Wikström, 1996). De passif, le consommateur devient davantage acteur, on parle alors dans le jargon marketing de " consommacteur».

Les années 2000 sont marquées par l'explosion des médias numériques. Parallèlement à l'évolution de l'internet vers les réseaux sociaux, le comportement du consommateur s'est modifié. Mieux informé, il est devenu un expert, bien plus difficile à manier. D'acteur, il devient « auteur ", créant des contenus multimédias, commentant et donnant son avis. $\mathrm{Du}$ « consomm'acteur » au « consomm'auteur », un « effet d'époque » est alors franchi. Bahuaud et Pecolo (2010) montrent que «l'effet d'époque» doit être inter-relié à « l'effet d'âge » et « l'effet générationnel » dont nous allons opérer une lecture allagmatique à partir des travaux de Carayol (2004). Cet auteur a montré que les organisations, quelles qu'elles soient, sont perpétuellement aux prises de processus de transformation, d'actualisation et de modulation, traversés par des mouvements antagonistes, identifiant quatre principaux processus de communication : chronogenèse, ordogenèse, échogenèse et morphogenèse. L'ensemble de ces contradictions pourrait partiellement 
s'expliquer par ce que Le Moënne (2008) nomme « une crise de l'Entreprise » comme institution, résultat des délocalisations massives rendant sa visibilité sur la scène sociale de plus en plus virtuelle.

L'entreprise entre alors dans un processus de chronogenèse, entre effet de virtualisation et effet de matérialisation, dans l'appréhension de son espace-temps, reflet de conjonctures sociales, économiques, politiques. Ces « effets d'époque » fonctionnent comme des marqueurs générationnels sources d'oppositions inter-générations. Bien que co-existantes, la " culture des pairs » tend à se substituer à celle des "pères ", les liens intragénérationnels se renforcent, forment des tribus, tandis que dans le même temps des similitudes autour de valeurs intergénérationnelles tendent à réduire la fracture entre «jeunes» et «seniors» (Bahuaud et Pecolo, 2010). Tout se passe comme si des processus de configuration et d'ordonnancement tendaient à modeler ces rapports d'interaction, soit dans le sens de la coopération et de la participation (liaison intergénérationnelle), soit dans le sens de l'émancipation et de l'indépendance (autonomie générationnelle). De tels processus peuvent alors être qualifiés d'ordogenèse, entre symétrie (logique horizontale) et asymétrie (logique verticale) des pratiques de communication. Cette mise en tension frappe en première ligne les sphères managériales, formant le terrain d'une échogenèse. Celles-ci sont alors en forte demande de nouvelles références à des valeurs, ce qui conduit à nourrir les discours d'entreprises d'une charge symbolique de plus en plus forte, en marge des logiques argumentatives traditionnelles. Les critères de segmentation par tranche d'âges élaborés par les professionnels du marketing s'affinent toujours plus pour tenter de cerner un " effet d'âge " qui serait a priori caractérisé par des usages précis circonscrits à un âge spécifique. Cependant, cet «effet d'âge " doit être mis en perspective avec un "effet générationnel ", d'individus ayant été jeunes à un même moment, explicatif d'habitudes, d'attentes, de comportements hérités dans la jeunesse et qui perdurent à l'âge adulte. C'est pourquoi à la variable âge, est désormais associée celle de " socio style ", c'est-à-dire un mode de vie associé à des valeurs spécifiques qui se répercutent sur les pratiques de consommation. Les sociostyles donnent lieu à une prolifération de dénominations, dont par exemple la typologie anglo saxonne de Strauss et Howe (1991) ou encore celle française d'Excousseau (2000) : 


\begin{tabular}{|c|c|}
\hline $\begin{array}{l}\text { Génération « héritage » } \\
\text { avant } 1941 \\
\text { (Génération « silencieuse ») }\end{array}$ & $\begin{array}{l}\text { Prêts à souffrir pour mériter leur gratification. Marqués par } \\
\text { la guerre, connaissent le manque. Moins branchés sur les } \\
\text { technologies de l'information. Dociles à l'égard de l'autorité. Ont } \\
\text { des obligations morales fortes et un sens du devoir prononcé. }\end{array}$ \\
\hline $\begin{array}{l}\text { Génération « Naturel » } \\
\text { 1941-1968 } \\
\text { (Génération «baby-boom») }\end{array}$ & $\begin{array}{l}\text { La vie, c'est le travail ; la réussite se mesure par la carrière. Sont } \\
\text { préoccupés par la famille, mais avec le plus haut taux de divorce } \\
\text { jamais vu. Déchirés par les valeurs parentales et la satisfaction } \\
\text { du « moi ». Pouvoir se procurer de belles choses. Ont inventé la } \\
\text { consommation et inculqué la surconsommation aux générations } \\
\text { suivantes. Davantage de choix de vie. }\end{array}$ \\
\hline $\begin{array}{l}\text { Génération « Réseaux » } \\
\text { 1969-1977 } \\
\text { (Génération «X») }\end{array}$ & $\begin{array}{l}\text { Plutôt égocentriques, cyniques et sarcastiques. Recherchent la } \\
\text { gratification immédiate de leurs besoins. Aiment expérimenter. } \\
\text { Critiquent et remettent en question l'autorité. Vivent dans le } \\
\text { présent et pour le présent. La vie, ce n'est pas juste le travail. }\end{array}$ \\
\hline $\begin{array}{l}\text { Générations « mosaïque " } \\
\text { À partir de } 1977 \text { (avec un } \\
\text { palier depuis } 11 / 09 / 01 \text { et un } \\
\text { possible retour d'une nouvelle } \\
\text { "génération silencieuse ») } \\
\text { (Génération «Y } \text { ") }\end{array}$ & $\begin{array}{l}\text { Génération de l'internet. Plutôt individualistes, portés à } \\
\text { l'hypersexualisation. Accordent plus de valeur au matériel, moins } \\
\text { de valeur aux règles, rebelles. Ont une conscience mondiale très } \\
\text { présente. Très à l'aise avec les technologies. Précoces : essaient } \\
\text { plusieurs choses très vite. }\end{array}$ \\
\hline
\end{tabular}

Figure 1 : Tableau descriptif de chacune des quatre générations (Strauss \& Howe, 1997, et Excousseau, 2000) inspiré des travaux de Lorey, Cailleba, 2010)

On observe des organisations désormais aux prises d' « injonction paradoxale », entre logique de marché personnalisée de type «one-to-one» et logique de marché « communautaire »: le consommateur attend de la marque qu'elle le considère comme individu singulier tout en lui offrant de rejoindre une communauté d'appartenance, avec laquelle il veut entrer en affinité sans pour autant y être amalgamé. Cet «effet générationnel » touche la génération des $\mathrm{X}$ mais surtout celle des $\mathrm{Y}$, familiarisée très tôt dans leur vie, avec les technologies numériques. Il constitue un processus de morphogenèse, expliquant notamment la démultiplication sur l'internet, de campagnes de marketing participatives sous la forme « d'appel à contribution».

\section{L'étude de cas Danette : une méthodologie qualitative d'analyse de contenu de la communication numérique de la marque}

Pour étayer notre propos, nous avons retenu une méthodologie d'inspiration qualitative fondée sur l'analyse d'une étude de cas, à partir d'un corpus documentaire (Delcambre, 1999) composé de récits discursifs hagiographiques issus du site internet de la marque Danette (www.danette.fr). « La méthode de l'étude de cas consiste donc à rapporter une situation réelle prise dans son contexte, et à l'analyser pour voir comment se manifestent et évoluent les phénomènes auxquels le chercheur s'intéresse " (Mucchielli, 1996). Selon 
Babbie (1985, cité dans Mucchielli, 1996), l'objectif suivi n'est pas une représentativité statistique du phénomène étudié mais un enrichissement de théories existantes, dans deux démarches possibles d'analyse : inductive ou déductive. La première consiste à repérer au sein de l'étude de cas, des patterns, des récurrences, pour construire pas à pas une théorie.

La deuxième, la démarche déductive, est celle dans laquelle nous nous inscrivons ici, où, à partir d'une sélection de certains matériaux du cas, nous allons chercher à montrer la dimension explicative, confirmative, de modèles théoriques déjà construits par d'autres chercheurs. Ainsi nous optons pour une étude de cas instrumentale, parmi les trois types (intrinsèque, instrumental, multiple) mis en évidence par Mucchielli (1996, citant Stake, 1994), " particulièrement appropriée dans les situations où le chercheur veut illustrer des phénomènes préalablement définis dans un modèle théorique ». Notre démarche n'étant pas inductive, nous avons volontairement exclu l'approche par études de cas multiples, laquelle s'inscrit dans une perspective de comparaison de résultats entre plusieurs situations afin d'en dégager les récurrences constitutives de l'élaboration graduelle d'une théorie.

Nous avons choisi le cas de la marque Danette car celle-ci constitue une marque emblématique dans l'imaginaire des Français depuis sa création en 1970, grâce à une communication qui s'est appuyée, dès l'origine, sur les liens intergénérationnels des consommateurs potentiels. En outre, elle a fait preuve d'innovation constante dans la création de recettes originales pour renouveler les saveurs commercialisées. Elle fait également partie des marques qui ont investi l'internet dès 2006, dans une optique de communication numérique participative. Sur le segment de la crème dessert, elle reste la seule marque avec un tel positionnement dans l'imaginaire collectif, dont les recettes et l'emballage sont désormais copiés par d'autres marques dont notamment celles de distributeurs ${ }^{2}$. Aucune autre marque n'est venue faire preuve d'innovation de rupture sur ces gammes de produits. Dans d'autres univers de produits de grande consommation, de plus en plus de marques s'essayent, ces dernières années, au marketing générationnel (Kickers, Nutella, Kit-Kat, Cadum...). Nous avons choisi de ne pas étudier leurs cas car, d'un point de vue chronologique, ils ne nous paraissaient pas directement comparables à celui de Danette, s'agissant d'un repositionnement opportuniste somme toute récent, essentiellement centré d'ailleurs, sur une communication de type transgénérationnel. Le cas Danette présente l'avantage, outre un positionnement générationnel historique, de montrer une évolution d'une communication médiatique, télévisuelle, intergénérationnelle à une communication numérique transgénérationnelle.

2. Depuis son lancement en 1970, 13,1 milliards de pots de 125 grammes ont été vendus en France. 22 pots de Danette sont vendus chaque seconde dans l'Hexagone. Danette est consommée au moins une fois par an par plus d'un foyer français sur deux. Chaque foyer achète une Danette 5 fois par an en moyenne. in Journal du net, [En ligne. Page consultée le 3 juin 2010]. Disponibilité et accès http://www.journaldunet.com 
La constitution de l'étude de cas Danette s'est faite selon la pratique préconisée par Mucchielli (1996) en deux phases : tout d'abord celle de cueillette et de mise en forme des informations, puis celle d'analyse du cas. La première consiste en la description du cas lui-même, à partir de laquelle on procède à une analyse de contenu théorisante afin de monter en niveau d'abstraction à partir d'une sélection des éléments utiles du cas. Nous nous sommes focalisée sur le site internet de la marque Danette (www.danette.fr ; cf. figure 2).

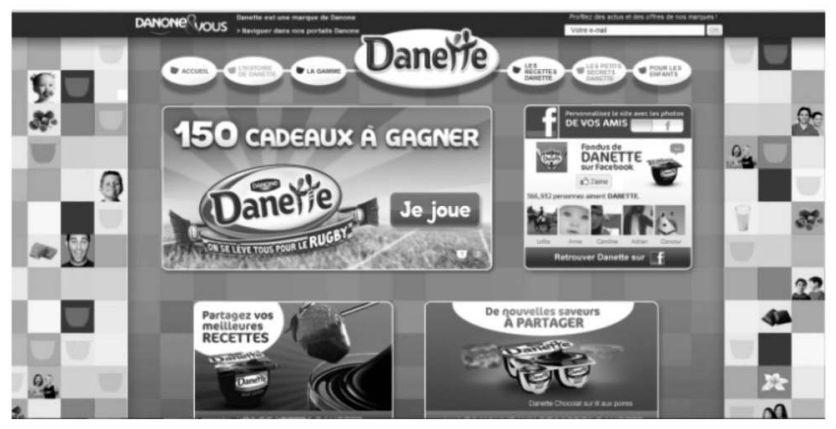

Figure 2 : Page d'accueil du site www.danette.fr

Ce site comporte six rubriques principales :

- Accueil : il s'agit de la page présentant les six rubriques et quatre zones d'actualités commerciales défilantes dans un diaporama dans la partie centrale de la page (un jeu, le lien Facebook, le partage de recette, le lancement de nouvelles saveurs, la tournée des 40 ans).

- L'histoire de Danette : cinq décennies d'évolution de la marque sont racontées de 1970 à 2010, chacune d'elle faisant l'objet d'une sous-rubrique.

- La gamme : présentation des vingt-six produits de la gamme Danette à partir de laquelle l'internaute a la possibilité de filtrer les produits avec les critères de "nouveauté ", « saveurs ", « textures ".

- Les recettes Danette : suggestions de recettes à base de produits Danette. En outre, les internautes peuvent adresser leur recette à la marque en remplissant les champs concernés (coordonnées, liste des ingrédients, descriptif de la préparation) à partir de cette rubrique et joindre une photo de cette recette.

- Les petits secrets Danette : cette rubrique se subdivise en huit sousrubriques dont quatre sur la traçabilité des principaux ingrédients : « de la vache au pot de Danette ", "Secret du pot ", «+70\% de lait ", " Du bon chocolat ». Deux sous-rubriques sont en lien avec l'historique de la marque " 40 ans qu'on se lève tous pour Danette » et " Découvrez son créateur ». Enfin la dernière (« Partagez vos souvenirs Danette») renvoie explicitement 
vers la page Facebook de la marque pour que les internautes y laissent des commentaires.

- Pour les enfants : cette rubrique est subdivisée en trois sous-rubriques : "Coloriages ", «Découpages », «Danette et toi ». Il est à noter qu'aucun de ces trois liens ne fonctionnent.

Chaque année depuis 2006, Danette renouvelle, à la fois sur son site et sur sa page Facebook, un appel au vote par lequel les internautes sont invités à élire la nouvelle saveur qui sera commercialisée.

Parmi ces rubriques, nous avons réalisé une codification dans le cadre de l'analyse de contenu (Bardin, 2007), c'est-à-dire une caractérisation du sens à partir de la réalité exprimée dans le cas. Nous avons ainsi retenu, plus particulièrement, les rubriques et sous-rubriques racontant l'histoire de la marque afin de mettre en exergue les évolutions en termes de communication intergénérationnelle. Concernant les liens sur la zone centrale de la page d'accueil, nous avons analysé ceux ayant trait au renvoi vers le réseau social Facebook, et le lien vers la tournée "Chez Danette ", de mai à juillet 2010, dans les villes de France, pour les 40 ans de la marque, mieux à même de rendre compte du passage de la communication intergénérationnelle à une communication davantage transgénérationnelle. Les meilleurs moments de cette tournée sont également accessibles par un lien sur la page Facebook de la marque.

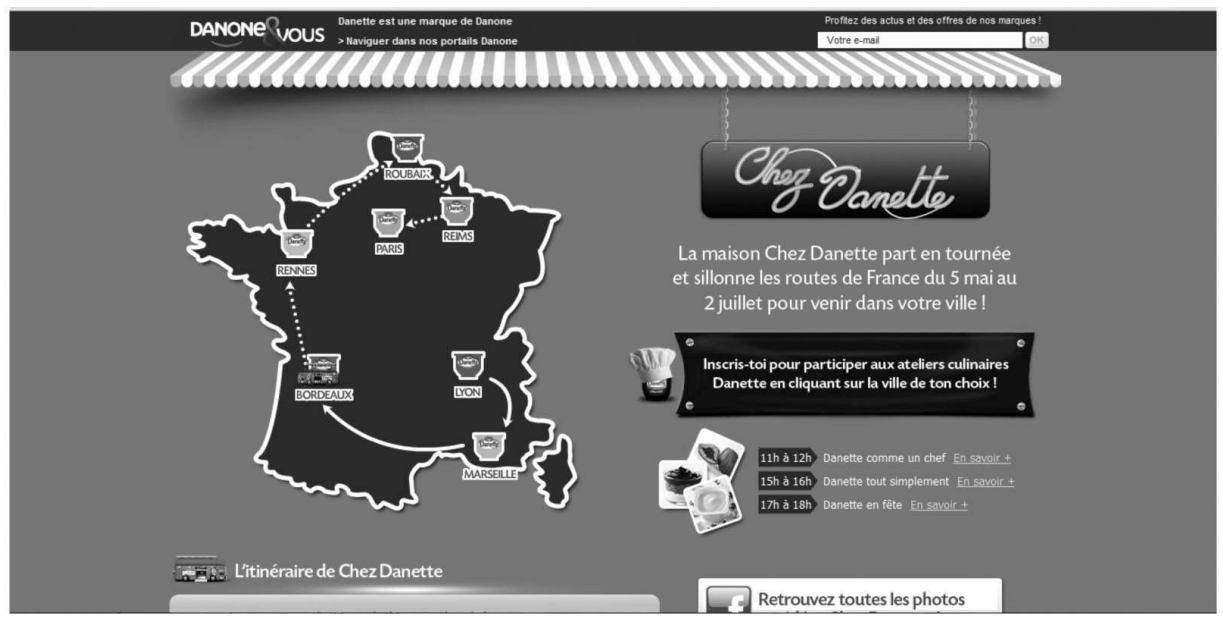

Figure 3 : Page d'accueil de la rubrique concernant la tournée nationale "Chez Danette " 


\section{$\mathrm{C} \& O \mathrm{n}^{\circ} 40$}

Trois ateliers culinaires thématiques sont proposés au public avec comme ciblage clairement affiché « les adultes » ou « la famille ». Pour participer, il est préférable de s'inscrire via la page Facebook afin d'éviter des temps d'attente et bénéficier en remerciement d'un cadeau.
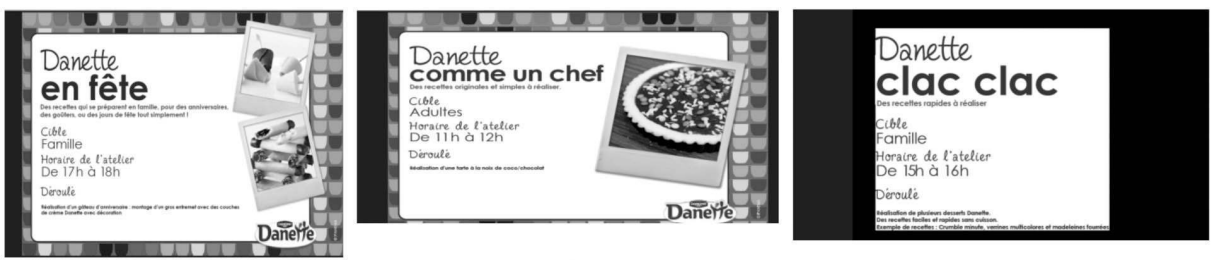

Figure 4 : Descriptif des trois ateliers proposés lors des villes visitées par la tournée " Chez Danette»

Enfin et en complément, du 18 au 26 septembre 2010, Danette ouvre un espace éphémère à Paris "Chez Danette ». Dans ce bar, les consommateurs sont invités à déguster les seize parfums de la gamme Danette qu'ils pourront agrémenter de fruits, céréales etc. En outre, une exposition retraçant l'histoire de la marque depuis 1970 est proposée ainsi que des leçons de cuisine à base bien sûr de crème dessert Danette. En parallèle, la marque crée un livre sur le thème «Danette, 40 ans, 40 recettes » à base de produits Danette. Pour cet anniversaire, Danette lance également l'opération «Souriez, vous êtes une Danette » par laquelle les internautes peuvent envoyer une photo pour figurer sur le pot Danette de leur parfum préféré.

Nous avons, ainsi, laissé volontairement de côté, les rubriques concernant les descriptifs des gammes de produits et des secrets Danette au sujet des ingrédients, car il nous apportait peu pour la problématique de cet article.

A partir de cette codification, nous avons ensuite conduit un travail de catégorisation et de mise en relation afin de confronter nos choix théoriques à cette étude empirique. L'analyse théorique nous emmène dans deux grandes directions qui feront chacune l'objet d'une partie spécifique. Tout d'abord, l'imaginaire sociotechnique tel que défini par Flichy (2001) nous servira de cadre d'analyse théorique pour expliquer chacune des cinq décennies (1970, 1980, 1990, 2000, 2010) présentées dans la rubrique «L'histoire de Danette ». Sur le site internet, un titre différent résume l'actualité forte de la marque correspondant à la décennie concernée (exemple : «1970: Danette arrive sur la table des Français »). Cet imaginaire inclut un collectif d'acteurs, concepteurs et usagers, dont les représentations symboliques s'expriment par des discours.

Ensuite, nous avons relevé une forte dimension participative par laquelle Danette demande aux internautes de participer à un ensemble d'actions en s'inscrivant et votant sur le site internet et la page Facebook de la marque (l'envoi de la recette dans la rubrique "Recette Danette ", l'opération «Souriez vous êtes une Danette »; la tournée « Chez Danette »; le vote 


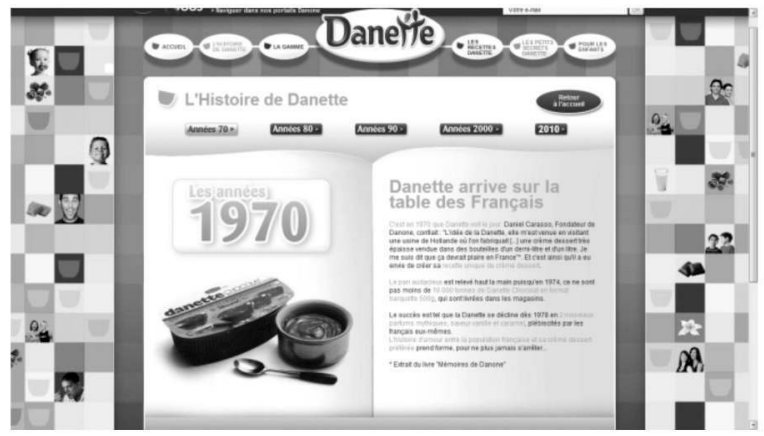

Figure 5 : Exemple de l'historique de la marque

en ligne annuel depuis 2006 pour élire le futur parfum commercialisé par la marque). Les travaux de Dujarier (2008) nous permettront d'éclairer ces résultats sous l'angle du " travail du consommateur ", dans le sens d'une " co-création marque-consommateur ». Enfin, nous discuterons l'ensemble de ces résultats par une analyse théorique allagmatique des publics visés par Danette sous l'angle de la communication générationnelle en mettant en évidence les segments de consommateurs visés (Bahuaud et al., 2010, Carlutti, 2004, Tréguer et al., 2005).

\section{Du " On se lève tous pour Danette " à " On vote tous pour Danette " : un imaginaire sociotechnique du lien transgénérationnel}

L'évolution du slogan de «On se lève tous pour Danette » à «On vote tous pour Danette " résume à elle seule l'évolution de l'histoire de la marque mise en récit dans la rubrique "L'histoire de Danette ». Elle révèle un imaginaire collectif autour de cette marque, revendiqué d'ailleurs au cours d'un micro-trottoir interviewant des consommateurs et différents salariés de Danette, valorisé dans une vidéo présente dans la rubrique «Les secrets de Danette, 40 ans qu'on se lève tous pour Danette ». Les théoriciens des marques ont trouvé dans le Storytelling (Salmon, 2007), l'outil qui leur permet de refléter (sinon de créer) une culture et des valeurs partagées par l'entreprise au travers d'une histoire fédératrice. Dans leur article sur le renouveau à apporter aux marques, Burnet et Hutton (2007) précisent que l'histoire de l'entreprise doit particulièrement mettre en valeur des éléments propres à impliquer le client. L'imaginaire sociotechnique (Flichy, 2001) montre un circuit (cf. figure 6) suivi par l'innovation dont les phases sont constituées non seulement, par des évolutions techniques, mais aussi par des discours dans un registre lui-même évolutif, entre l'utopie et l'idéologie. Ce sont ces discours qui permettent, ou non, à l'innovation de franchir des phases sociotechniques (objet-frontière, objet-valise, verrouillage technologique), qui feront que l'innovation sera diffusée ou abandonnée. 


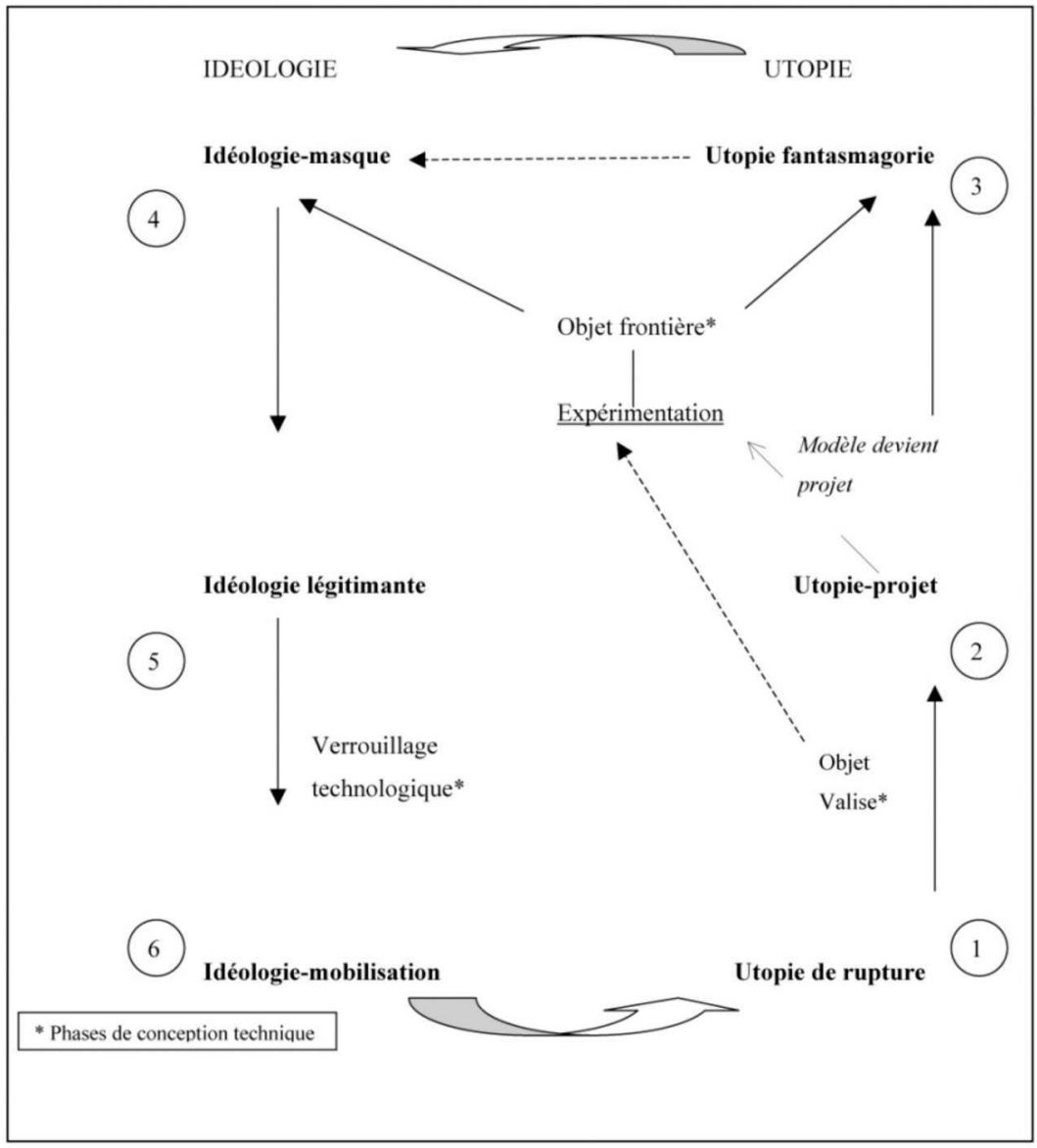

Figure 6 : L'imaginaire sociotechnique (Flichy, 2001)

Ces discours qui soutiennent ou empêchent l'innovation, empruntent au registre discursif, à la fois, du "récit témoignage » et du "récit d'influence » tels que définis par Boudes (2006). Le récit témoignage peut se définir comme l'art de raconter un événement à quelqu'un qui n'y a pas participé ; il relate une expérience vécue, il fait partie des registres discursifs omniprésents au sein des organisations. Dans le cas Danette, pour assoir ce récit, l'internaute peut aussi visionner une interview du fondateur en format vidéo (sous-rubrique «Découvrez son créateur » dans les « secrets de Danette »).

Le « récit d'influence», quant à lui, a pour vocation de persuader en cherchant à glisser un certain point de vue à partir d'informations sélectionnées pour orienter l'interprétation du destinataire. Lidée est de mettre en comparaison le début du récit avec sa fin pour montrer ce qui a changé grâce à un événement de rupture en milieu de récit, et ce, pour en tirer une morale finale. 
Ces récits mixtes entre témoignage et influence peuvent être qualifiés de scripts (Boudes, 2006), en ce qu'ils décrivent, linéairement et sans intrigue, des modes de vie et des modes de production industriels, avec l'intention incitatrice de poser une norme d'équité à la fois organisationnelle et consumériste. Cette linéarité est fortement perceptible par la structure de la rubrique "L'histoire de Danette » en cinq sous-rubriques chacune correspondant à une décennie, que nous relions aux différentes phases identifiées par Flichy (2001).

Au cours de la décennie 1970, "Danette arrive sur la table des Français ». Ce discours utopique est celui qui va permettre aux innovateurs de laisser s'exprimer toutes leurs idées, c'est un bouillonnement de créativité. Le fondateur de Danone, Daniel Carusso explique que l'idée de fabriquer la première crème dessert Danette lui est venue suite à la visite d'une laiterie-crèmerie en Hollande où une crème dessert épaisse était fabriquée et conditionnée dans des bouteilles d'un demi-litre. La phase 1, l'utopie de rupture, correspond au moment où une innovation rencontre d'autres mondes sociaux : ceux d'autres concepteurs avec des innovations concurrentes, ceux des usagers dont on ne sait encore quel accueil ils réserveront au nouveau produit. L'étape technique associée est celle de l'objet-valise, un peu brouillonne, féconde, riche en nouvelles idées et possibilités. C'est ainsi qu'en 1974 est née la première version des produits Danette, une barquette de 500 grammes au chocolat, qui vise la famille, parents et enfants, suivie quatre ans plus tard par deux nouveaux parfums, vanille et caramel. Ce stade peut se solder par l'abandon de l'idée qui devient une utopie-fantasmagorie, ou alors, poursuivre son évolution en passant à la phase suivante.

À partir des années 1980, Danette parvient à la deuxième phase, l'utopie-projet, une phase où l'innovateur va concrétiser son idée sous la forme d'une première expérimentation technique. L'étape technique associée à la phase d'expérimentation est celle de l'objet-frontière caractérisé ici par la poursuite du lancement de nouvelles saveurs mais aussi de nouvelles textures (Danette Crousti au riz soufflé ou aux billes colorées). Ce dernier signifie que l'innovation entre en confrontation avec d'autres techniques et d'autres acteurs. Pour qu'elle continue d'exister, son innovateur devra élaborer un compromis pour associer des partenaires divers. Les usages commencent à se construire, aidés par un discours qui revendique la nouveauté, l'exemplarité, l'apport social de l'innovation, concrétisé par le démarrage de la célèbre saga publicitaire télévisée «On se lève tous pour Danette ». Cette saga intergénérationnelle met en scène plusieurs générations au sein de la famille, les grands-parents, les parents baby-boomers et leurs enfants, les X.

D'utopique, le discours devient idéologique : il s'agit de promouvoir l'innovation quitte à en masquer certaines facettes si besoin est, d'où la terminologie d'idéologie-masque à la quatrième phase. Danette y entre dans la décennie 1990 titrée : «La révolution des saveurs : à chacun sa Danette ». La marque entame une segmentation à «effet d'âge » plus marqué en termes 
de diversification produits à destination d'une part des adultes (Danette double saveur) et d'autre part des enfants (Danette Goûter au lait en pot de 70 grammes, format plus adapté à eux).

La cinquième phase marque une solidification toujours plus grande de l'innovation, un véritable « verrouillage technologique » où toutes les autres alternatives qui avaient pu être explorées aux phases précédentes, sont abandonnées. Il s'agit de légitimer l'innovation, d'où un discours à l'œuvre, que Flichy qualifie d'idéologie légitimante. «On vote tous pour la Danette des Français ». Danette poursuit le lancement de nouvelles saveurs et déploie une stratégie marketing sur les médias numériques. Ces générations d'enfants des décennies 1970 à 1980 sont aujourd'hui des adultes, rompus à l'utilisation des médias numériques. Pour capitaliser sur la proximité affective historique avec cette deuxième génération de consommateurs, Danone investit alors le Web en 2006, déployant un marketing transgénérationnel. La marque soumet trois nouveaux parfums au vote des internautes afin que ce soit les consommateurs qui décident du futur parfum commercialisé (celui ayant recueilli le plus grand nombre de suffrages). Le nombre d'internautes votants s'accroît d'année en année.

Par là-même, la sixième et dernière phase qui découle directement de la précédente correspond à une mobilisation maximale de l'ensemble des acteurs sociaux (concepteurs, usagers, médias etc.) pour assurer la légitimité de l'innovation en vue d'une diffusion large, d'où le qualificatif d'idéologie-mobilisation. En 2010, Danette fête ses 40 ans à travers un dispositif pluri médias (médias de masse, médias numériques, hors média) sous la bannière " On se lève tous pour les 40 ans de Danette ». Le clin d'œil transgénérationnel est évident avec la reprise du célèbre " On se lève tous... » associé au "relancement » de la barquette familiale d'origine. La marque assoit son concept « La Danette des Français " par le recours à un large appel à contribution des internautes " petits " et " grands ». Nous allons analyser plus finement dans la partie suivante ce dispositif qui a atteint le million de participants.

\section{La " Danette des Français " : l'appel à contribution d'une génération coproductrice de sa consommation}

En 2006, Danette se lance dans la communication numérique en créant l'opération marketing «La Danette des Français ». Elle demande aux internautes de voter sur le site internet pour leur parfum préféré après avoir goûté deux nouveaux parfums. Celui qui recueille le plus de voix restera commercialisé dans les supermarchés. Cette opération est reconduite chaque année et voit son nombre de votants augmenter, passant de 400000 en 2006 à 1,4 million dès 2007 et dépassant les 2 millions à partir de 2008 (parmi les parfums plébiscités, on trouve brownie, chocolat-coco, crème brulée ou encore chocolat blanc). En 2011, ce concours oppose des régions à travers 
les saveurs à élire : caramel au beurre salé pour la Bretagne, spéculos pour la région parisienne et nougat-miel pour le Sud. En 2009, Danette crée sa page Facebook et renforce les actions participatives. Il est à noter que la marque est également présente sur le réseau social Twitter mais nous ne l'intégrons pas dans notre analyse car, d'une part, le site internet n'en fait pas mention, et d'autre part, la marque possède seulement 172 abonnés et la plupart de ses derniers tweets renvoient explicitement les internautes vers la page Facebook, qui, elle, totalise plus de 707000 amis

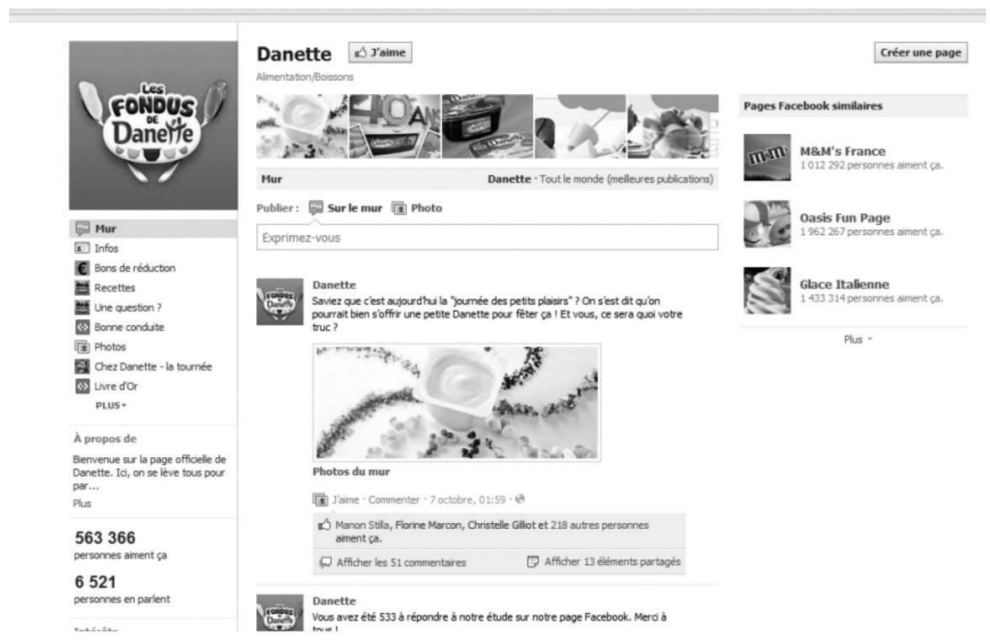

Figure 7 : Extrait de la page Facebook de Danette (7/10/11) : "Saviez-vous que c'est aujourd'hui la journée des petits plaisirs ? "

Au-delà des simples commentaires que les internautes peuvent laisser sur la page Facebook, la marque propose un dispositif participatif dans plusieurs directions. Tout d'abord, concernant l'opération «La Danette des Français », les internautes peuvent voter sur Facebook ; le succès est tel qu'en 2011, 260000 internautes supplémentaires sont devenus fans de la marque. Les internautes peuvent consulter et partager des recettes de cuisine. Pour les 40 ans de Danette en 2010, les internautes peuvent s'inscrire sur Facebook pour participer aux ateliers de la tournée nationale Danette dans les principales villes de France. Toujours dans le cadre de cet anniversaire, le réseau social a aussi servi de relai pour l'opération "Souriez vous êtes une Danette » qui propose aux fans de la marque de faire partie des 480 premiers internautes à déposer une photo d'eux sur un site événementiel dédié (www.souriez. danette.fr, qui n'existe plus à ce jour) afin de personnaliser les pots de 500 grammes de crème dessert (à titre informatif 40000 internautes ont participé à cette dernière opération). 
Depuis 2007, la plupart des grands groupes industriels (Mac Donald, Kinder, Renault, Danone, Nestlé, Ferrero, Unilever, etc.) ont mis en œuvre des campagnes participatives du même type que Danette qui sont qualifiées d'appel à contribution. Reniou (2009) propose une « théorie de la participation aux opérations participatives des marques " comme un «système d'échanges interconnectés entre plusieurs intervenants : la marque, les participants et les non-participants ». Ainsi ces opérations se caractérisent par leur accessibilité au plus grand nombre, leur caractère public (tout le monde peut participer ou consulter la participation des autres) et agissent comme une médiation entre la marque et ses consommateurs. Elles peuvent donner lieu ou non à une rétribution du participant. Si rétribution il y a, celle-ci prend la forme d'une somme d'argent, d'un cadeau ou de la médiatisation du participant sur un support (photo sur l'emballage d'un produit, acteur d'une publicité diffusée sur un média de masse etc.). Cependant, il existe une forte incertitude quant à la manière dont les individus sollicités s'approprient ce type d'opérations et leur investissement dans la participation. C'est pourquoi la promotion de ces opérations passe par une forme de communication dite virale, un bouche-àoreille revisité grâce aux technologies numériques.

Dans une telle perspective, Dujarier (2008) avance l'idée de «travail du consommateur ", dans la continuité des travaux interactionnistes conduits par l'École de Chicago sur la " relation de service », et des recherches en marketing sur la « servuction » (Eiglier, Langeard, 1987), pour désigner la participation du client à l'élaboration d'un service. Parmi les trois formes de «travail du consommateur » mis en évidence par l'auteur, celle déployée par Danette s'apparente à de la « coproduction collaborative ». La marque demande aux internautes de produire bénévolement des contenus en ligne en faisant appel à leur créativité, leur savoir-faire et intelligence. C'est une forme d'externalisation récente issue du développement des réseaux socionumériques sur l'internet, de type crowdsourcing. A travers l'internaute, c'est le consommateur qui est visé, et qui témoigne d'ailleurs d'un certain enthousiasme à livrer des informations et des créations de divers types que les entreprises récupèrent à des fins d'extensions marchandes de leur marché. En outre, ce travail du consommateur est renforcé par le modèle économique des réseaux socionumériques eux-mêmes, puisque celui-ci est basé sur une injonction de la prescription généralisée, laquelle articule l'individuel au collectif, par le truchement des contraintes de fonctionnement imposées par les standards du logiciel sous-tendant le réseau. Stenger (2011) postule qu'une prescription individuelle porte en elle le potentiel d'une prescription collective pour les membres du réseau.

À travers cette stratégie participative, la marque vise un « effet transgénérationel » des tribus des $\mathrm{X}$ et des $\mathrm{Y}$. "Les communautés de consommateurs ont trouvé avec internet des moyens de se regrouper et de partager des conversations autour de l'objet de leur passion. Ils peuvent 
notamment y exister à travers la reconnaissance sociale de leur gratification à consommer qui se réalise à travers des discussions où l'affect et le plaisir prennent une place importante " (Cordelier, 2010). Danette fait partie des " marques aimées", qui ont su faire en sorte que «le lien importe plus que le bien " (Cova cité par Kabla et al., 2011). Elles adoptent alors un rôle d'accompagnement de leurs communautés virtuelles dans les médias sociaux, lesquelles aiment et défendent la marque. Cette analyse révèle un usage de l'appel à contribution numérique davantage comme support de « discours de la maisonnée » (D’Almeida, 2001) que comme véritable outil communautaire de démocratie participative en ligne. Ces discours par lesquels le sens prend corps, se présentent de manière horizontale par rapport à la verticalité de la communication institutionnelle, formelle. Ils viennent en appui à l'action, pour assurer la continuité et la consolidation. La logique communautaire qui préside à ces discours de la maisonnée tend à vouloir enrôler et impliquer le plus grand nombre possible d'acteurs.

\section{Des liens intergénérationnels à la transmission transgénérationnelle : une discussion allagmatique autour d'un marketing de la nostalgie}

Après avoir joué la carte familiale des relations intergénérationnelles des années 1980, Danette aborde un virage transgénérationnel en recourant aux médias numériques depuis 2006. La génération ainsi visée est technophile, née dans les décennies 1970 et 1980, hyperconnectée, que ce soit par l'intermédiaire de son ordinateur ou d'un smartphone. «Le numérique arrive donc à un moment où les personnes manifestent un fort besoin d'interactivité qui peut refléter un désir de considération, de participation à toutes les facettes de la vie sociale, économique et politique de la société dans laquelle elles se situent " (Debos, 2008). La moyenne d'âge des usagers du réseau social Facebook est de 37 ans (Kabla, Gourvennec, 2011).

La marque mise sur les enfants d'hier pour toucher aujourd'hui les " adulescents " (adultes, voire parents, régressifs sur leur enfance et notamment sur les produits emblématiques qui y sont liés) en jouant la carte de la nostalgie. Les frontières générationnelles sont brouillées pour se centrer sur des valeurs communes à toutes les générations. On parle alors de rétromarketing ou marketing de la mémoire; la marque puise dans les racines historiques pour créer un lien avec ses clients dans un processus d'échogenèse, dans un mouvement contradictoire entre un client considéré à la fois comme roi mais aussi au service de l'entreprise (Dujarier, 2008). Tout se passe comme s'il existait une forme de sécurisation derrière la nostalgie. D'ailleurs Danette fait revivre de nouveau la barquette familiale, symbole d'une rétro innovation. La marque se revendique en tant que " patrimoine émotionnel des Français " renforçant l'imaginaire sociotechnique identifié par Flichy (2001). Ainsi comme l'ont souligné Bahuaud et Pecolo (2010), en parallèle 
de segmentations par âge toujours plus précises, on assiste à un retour du « grand public » (La Danette des Français) qui témoigne d'une « confusion des âges ». En termes de pratiques de marketing générationnel, Tréguer et Ségati (2005) identifient six âges dans le cycle de vie d'un individu de la naissance à sa mort, par tranche de 15 années. La génération participative que nous évoquons se situe, sociodémographiquement, aux âges 2 et 3 qualifiés respectivement de « jeunesse » et de «nidification». Le premier se place dans l'instabilité de l'adolescence tandis que le deuxième correspond à la stabilité de l'entrée dans la vie adulte. Du point de vue des critères de personnalité, ces auteurs associent à ces catégories d'âges les sociostyles de Junior (0-25 ans) et de Major (25-50 ans). Les Juniors possèdent un fort pouvoir d'influence et de prescription des produits et services auprès de leurs proches. Les Majors se subdivisent en deux segments : Nester (25-34 ans) et Manager (35-49 ans) dont les points communs sont le manque d'argent, et une situation familiale mouvante. Dans une telle configuration, les Nesters construisent leur nid alors que les Managers gèrent l'acquis. « Loin du faux idéal du one-to-one, le marketing générationnel propose une approche médiane entre le marketing de masse et le marketing personnalisé : chaque individu de par ses âges (biologique, social...), de l'époque dans laquelle il vit et du cycle de vie dans lequel il se trouve, est unique mais reste en relation avec un groupe d'individus qui lui ressemble et qui vit la même chose que lui » (Carlutti, 2004).

Cependant, on remarque que les seniors, hier parents consommateurs de Danette dans les années 70 et 80 (génération des baby-boomers) tendent à être exclus des dispositifs communicationnels déployés par la marque Danette. La figure des grands-parents n'est plus présente dans les campagnes pour ne mettre en scène que de jeunes adultes ou des adultes parents de jeunes enfants. Les thématiques des campagnes d'appel à contribution sur l'internet s'adressent avant tout aux générations $\mathrm{X}$ et $\mathrm{Y}$ et finalement à une transmission transgénérationnelle réduite, pour ne pas parler de quasi cloisonnement générationnel en marge des effets de discours sur les liens intergénérationnels. Ces pratiques communicationnelles de prescription génèrent des processus de morphogénèse, soit dans une direction de distinction et de singularisation, soit dans une direction d'isomorphisme et d'identification à un collectif. Stenger (2011) et Proulx (2011) avancent l'idée d'une forme de mise au travail du contributeur de réseau socionumérique. Selon Stenger (2011), « on ne peut pas ne pas prescrire ». Les deux principales finalités de la prescription identifiées par l'auteur se trouvent être la « prescription de la consommation et des marques dans une logique marketing " et la prescription de "l'action collective sur les réseaux socionumériques ». Dans le cadre de la première forme de prescription, les contributeurs des réseaux socionumériques évoquent la marque et ses produits comme éléments de leur quotidien, tandis que la deuxième implique la publication de contenu par les utilisateurs (ce qui s'appelle dans le jargon de l'internet, l' User Generated Content-UGC). 
Par cette prescription numérique généralisée, c'est essentiellement la génération $X$ qui est concernée par ce " patrimoine émotionnel » lié au célèbre "On se lève tous pour Danette ». Elle fait découvrir la marque à la génération suivante, $Y$, qui n'a pas ou peu connu cette période phare, mais qui s'avère sensible à l'émotion procurée ("On se lève tous pour les 40 ans de Danette »). Dans cette perspective transmissive de génération en génération, pourquoi la marque développe-t-elle une quasi ségrégation à l'égard des parents de la génération $\mathrm{X}$ ? Est-ce pour éviter des risques liés aux regrets, aux frustrations et au retour à un passéisme ringard ? Tout se passe comme si un « effet d'époque » autour des pratiques des réseaux socionumériques agissait comme marqueur générationnel. En d'autres termes, un processus de chronogenèse se répercute sur un processus d'ordogenèse axé sur une " horizontalité des pairs ", faisant fi de la "verticalité des pères ». Le marketing transgénérationnel impose de demeurer en adéquation avec le présent, d'innover et de revisiter l'identité de la marque.

En conclusion, nous avons voulu retracer le parcours d'une marque spécifique, Danette, sur son choix d'une communication intergénérationnelle afin d'assurer la médiatisation de ses produits et ce, dès ses débuts (1970/1980). Le célèbre slogan « On se lève tous pour Danette » venait en appui de publicités télévisuelles montrant plusieurs générations au sein d'une même famille se mettre debout en entonnant ce chant. En outre, ce qui a conforté définitivement notre choix de cette marque, est l'évolution de son positionnement vers une communication de plus en plus transgénérationnelle au fur et mesure que Danette a investi les médias numériques (création de son site internet en 2006 et de sa page Facebook en 2009). Nous avons opté pour une méthodologie reposant sur la pratique de l'étude de cas selon une analyse de contenu théorisante. Nous avons obtenu deux grandes catégories de résultats. La première est issue de l'analyse de l'historique de la marque qui se revendique comme " patrimoine émotionnel des Français " que l'on a appréhendé du point de vue théorique par l'imaginaire sociotechnique de Flichy (2001) pour retracer et prouver la genèse d'une communication générationnelle depuis une «verticalité des pères» à une " horizontalité des pairs ». Chacune des cinq décennies vécues par la marque montre des innovations en termes de recettes, d'emballages, de campagnes marketing. Ces innovations sont soutenues par des discours de type storytelling, entre témoignage et influence, évoluant d'un registre de l'utopie à celui de l'idéologie pour aboutir à la mobilisation maximale de l'ensemble des acteurs autour de la marque (consommateurs, médias, salariés). Un imaginaire se cristallise autour de l'opération participative sur internet « La Danette des Français » et le slogan est modifié pour devenir «On vote tous pour Danette ». La marque se focalise sur des opérations participatives dès 2006, où chaque année, les internautes peuvent voter pour le nouveau parfum à commercialiser, ou 
encore soumettre leur photo sur un site internet dédié pour figurer sur les emballages de pots de 500 grammes de crème dessert à l'occasion des 40 ans de la marque. L'ancrage théorique que nous avons alors convoqué est celui de la mise en avant d'un « travail du consommateur » (Dujarier, 2008) sous la forme d'une coproduction collaborative faisant appel à la créativité des internautes et à leurs capacités de devenir auteurs de contenus sur les réseaux socionumériques, et plus particulièrement, la page Facebook de la marque, dont le fonctionnement est lui aussi basé sur un système de prescription généralisée (Stenger, 2011). On observe alors le passage d'une économie de la transaction à une économie de la relation. Cependant, selon Dujarier, ce travail du consommateur, bien que bénévole, représente un coût, comme pour l'évaluation monétaire du travail d'un salarié de l'entreprise. Cette économie de la relation présente alors tout de même une facette marchande avec une visée d'extension du marché de l'entreprise. Enfin nous avons discuté l'ensemble de ces résultats en reprenant le corpus théorique présenté dans la première partie sur une approche allagmatique (Carayol, 2004) des pratiques d'un marketing de la nostalgie déployé par Danette. Les quatre processus allagmatiques (échogenèse, chronogenèse, morphogenèse, ordogenèse) révèlent des mouvements de tensions, des contradictions sur la stratégie de segmentation des cibles visées par la marque. Tout se passe comme si Danette oscillait entre une segmentation intergénérationnelle de type « tout public » (Bahuaud et al., 2010) fédératrice sur la thématique " La Danette des Français ", et une segmentation centrée principalement sur la génération $\mathrm{X}$ (Excousseau, 2000) et par répercussion sur celle des $\mathrm{Y}$ en recourant aux réseaux socionumériques. Est mise en scène la carte de la nostalgie des adultes autrefois enfants consommateurs de Danette, et la génération suivante sensible aux émotions suscitées chez la génération antérieure. La génération des baby-boomers, parents des X, semble écartée, comme si l'atteinte de l'âge senior faisait prendre le risque d'un positionnement passéiste voire ringard.

\section{BIBLIOGRAPHIE}

BABBIE E-L, The practice of social research (fifth edition), Belmont, Wadsworth, 1985, $624 \mathrm{p}$.

BAHUAUD M., PECOLO A., "De la segmentation à la confusion des âges : stratégies marketing et approches générationnelles des publics ", Actes du $78^{e}$ congrès ACFAS, Culture et identité dans les communications marketing, Université de Montréal, 11 mai 2010, p. 74-82.

BARDIN L., L'analyse de contenu, Paris, PUF, 2007, 291 p.

BOUILLON J.-L., BOURDIN S., LONEUX C., " De la communication organisationnelle aux "approches communicationnelles" des organisations : glissement 
paradigmatique et migrations conceptuelles ", Communication E' organisation, 2007, n 31, p. 7-25.

BOUDES T., "Les attracteurs de la notion de récit dans les organisations et les rôles des managers ", dans SOULIER E. (dir.), Le storytelling, Paris, Hermès Lavoisier, 2006, 22 p.

BOUTAUD J.-J., Sémiotique et communication : du signe au sens, Paris, L'Harmattan, 1998,320 p.

BURNETT J., HUTTON B., " New consumers need new brands ", Journal of Product E Brand Management, 2007, Vol.16, n5, p. 342-347

CARAYOL V., Communication organisationnelle : une perspective allagmatique, Paris, L'Harmattan, 2004, 236 p.

CARLUTTI M.-L., « Le marketing générationnel, quels impacts sur la stratégie et le mix-marketing ? », 2004, Visionary Marketing [En ligne, page consultée le 15 septembre 2011]. Disponibilité et accès http://visionarymarketing.com

CORDELIER B., « Communauté virtuelle d'amateurs et positionnement de marque ; Ethnocentrisme et déclinaison de marque ", Actes du 78e congrès ACFAS, Culture et identité dans les communications marketing, Université de Montréal, 11 mai 2010, p. 83-93.

D'ALMEIDA N., Les promesses de la communication, Paris, PUF, 2001, 259 p.

DEBOS F., «Les relations numériques individus-marques ", Documents numériques, mai 2008, vol.10, n³-4, p. 63-79.

DEGENNE A., FORSÉ M., Les réseaux sociaux, Paris, Armand Colin, 1994, 296 p.

DELCAMBRE P., Les recherches en communication organisationnelle : concepts et théorisations, journées d'études Org\&co, 3-5 juin 1999, Aix-en-Provence

DUJARIER M.-A., Le travail du consommateur. De McDo à eBay : comment nous coproduisons ce que nous achetons, Paris, La découverte, 2008, 246 p.

EIGLIER P., LANGEARD E., Servuction, le marketing des services, Paris, McGraw-Hill, "Collection Stratégie et management », 1987, 205 p.

EXCOUSSEAU J.-L., La mosaïque des générations, Comprendre les sensibilités et les habitudes des Français, Paris, Editions d'Organisations, Les Échos Éditions, 2000, 350 p.

FLICHY P., L'imaginaire d'internet, Paris, La découverte, 2001, 276 p.

KABLA H., GOURVENNEC Y., Les médias sociaux expliqués à mon boss, Bluffy, Éditions Kawa, «Les fondamentaux du Web et du Marketing », 2011, 418 p.

LE MOËNNE C., «Ethiques et contextes organisationnels », MEI, 2008, n²9, p. 39-54

LOREY L., CAILlEBA P., « Le concept de génération : application aux représentations du vin en France sur quatre générations ", Recherche et Applications en Marketing, 2010, Vol 25, n³, numéro spécial « Marketing et génération », p. 1-42.

MUCCHIELLI A., Dictionnaire des méthodes qualitatives en sciences humaines et sociales, Paris, Armand Colin, 1996, 275 p.

PROULX S., KWOK CHOON M-J, « L'usage des réseaux socionumériques : une intériorisation douce et progressive du contrôle social ». Hermès, 2011, n 59, p. 105-111.

RENIOU F., Opérations participatives des marques : pourquoi et comment faire participer les consommateurs? De la compréhension des opérations participatives et des motivations des 
consommateurs à s'y engager à l'analyse de leurs effets sur la marque. Thèse de Sciences de Gestion, Université de Paris Dauphine, 2009, 395 p.

SALMON C., Storytelling. La machine à fabriquer des bistoires et à formater les esprits, Paris, La Découverte, 2007, 247 p.

SOULIER E., (dir.), Le Storytelling : concepts, outils et applications, Paris, Hermès Lavoisier, 2006, 410 p.

STAKE R-E, "Cases studies ", dans Denzin, N.K et Lincoln, Y.S. (dir.), Handbook of qualitative research, London, Sage Publications, 1994

STENGER T., "La prescription de l'action collective ", Hermès, 2011, n59, p. 127-133.

STENGER T., COUTANT A., (dir.), « Ces réseaux numériques dits sociaux. Introduction ", Hermès, 2011, n59, 253 p.

STRAUSS W., HOWE N., Generations. The History of America's Future, 1584 to 2069, New York, Quill William Morrow, 1991, 544 p.

TREGUER J.-P., SEGATI J-M., Marketing générationnel, gay marketing, marketing ethnique, Paris, Dunod, 2005, 252 p.

WASSERMAN S., FAUST K., Social Network Analysis : Methods and Applications, England and New York, Cambridge University Press, 1994, 857 p.

WIKSTRÖM S., "The customer as a co-producer », European Journal of Marketing, 1996, n³0, p. 359-374.

\section{WEBOGRAPHIE :}

Site internet de Danette. Disponibilité et accès http://www.danette.fr

Page Facebook de Danette. Disponibilité et accèshttp://www.facebook.com/danette. france

Journal du Net. Disponibilité et accès http://www.journaldunet.com

Résumé : À partir de l'étude du cas Danette, nous voulons montrer la transition allagmatique d'une génération à l'autre, comment cette communication médiatique a engendré un basculement d'une "verticalité des pères "(On se lève tous pour Danette) à une "horizontalité des pairs"(On vote tous pour Danette). À travers cette question générationnelle, se pose une évolution d'une économie de la transaction à une économie de la relation, où pour demeurer compétitives, les entreprises doivent considérer le « cycle de vie client » plutôt que le « cycle de vie produit ». L'avantage concurrentiel de Danone se jouera sur un "ré-enchantement » consumériste, par la mise en œuvre d'une communication transgénérationnelle où, dans une démarche de co-création, le consommateur est étroitement associé à la commercialisation des produits et à la médiatisation de la marque. Danette construit alors rétrospectivement un véritable imaginaire sociotechnique à partir de son histoire. L'internet soutient ce dispositif d'un marketing de la nostalgie amorcé en 2006 et qui atteint son point culminant en 2010 pour l'événement «On se lève tous pour les 40 ans de Danette ». 
Mots-clés : processus allagmatiques, imaginaire sociotechnique, liens intergénérationnels, transmission transgénérationnelle, médias sociaux participatifs, marketing de la nostalgie.

Abstract : From the Danette case study, we want to show the allagmatic transition one generation to another, how the media communication has led to a shift of "verticality of fathers" ("We get up all for Danette") to a" horizontality of peers" ("We all vote for Danette"). Through this generational issue, there is an evolution of an economy of the transaction to an economy of the relationship, in which to stay competitive, companies must consider "the customer lifecycle" rather than the "product lifecycle". The competitive advantage of Danone will be played on a "consumer re-enchantment", thanks to the implementation of a transgeneration communication, where in a co-creation process, the consumer is closely associated with the brand marketing and media coverage. Then Danette restrospectively constructs a real sociotechnical imaginary from its history. The internet supports this marketing of nostalgia device begun in 2006 and that peaks in 2010 for the event "We get up all for Danette 40 years old".

Keywords : allagmatic processes, sociotechnical imaginary, intergeneration relationships, transgeneration transmission, social participatory media, marketing of nostalgia. 
